\title{
Specific mitochondrial calcium overload induces mitochondrial fission in prostate cancer cells
}

\author{
ISMAIL KADDOUR-DJEBBAR ${ }^{1,2}$, VIVEK CHOUDHARY ${ }^{1,2}$, CRAIG BROOKS $^{2}$, TAGHREED GHAZALY ${ }^{1}$, \\ VIJAYABASKAR LAKSHMIKANTHAN ${ }^{1,2}$, ZHENG DONG $^{1,2}$ and M. VIJAY KUMAR ${ }^{1,2}$ \\ ${ }^{1}$ Charlie Norwood VA Medical Center and ${ }^{2}$ Medical College of Georgia, Augusta, GA, USA
}

Received December 23, 2009; Accepted January 26, 2010

DOI: 10.3892/ijo_00000629

\begin{abstract}
Mitochondria are structurally complex organelles that undergo fragmentation or fission in apoptotic cells. Mitochondrial fission requires the cytoplasmic dynamin-related protein, Drp1, which translocates to the mitochondria during apoptosis and interacts with the mitochondrial protein, Fis 1 . Finely tuned changes in cellular calcium modulate a variety of intracellular functions; in resting cells, the level of mitochondrial calcium is low, while it is higher during apoptosis. Mitochondria take up $\mathrm{Ca}^{2+}$ via the Uniporter and extrude it to the cytoplasm through the mitochondrial $\mathrm{Na}^{+} / \mathrm{Ca}^{2+}$ exchanger. Overload of $\mathrm{Ca}^{2+}$ in the mitochondria leads to their damage, affecting cellular function and survival. The mitochondrial $\mathrm{Na}^{+} / \mathrm{Ca}^{2+}$ exchanger was blocked by benzodiazepine, CGP37157 (CGP) leading to increased mitochondrial calcium and enhancing the apoptotic effects of TRAIL, TNF $\alpha$ related apoptosis inducing ligand. In the present study, we observed that increasing mitochondrial calcium induced mitochondrial fragmentation, which correlated with the presence of Drp1 at the mitochondria in CGP treated cells. Under these conditions, we observed interactions between Drp1 and Fis1. The importance of Drp1 in fragmentation was confirmed by transfection of dominant negative Drp1 construct. However, fragmentation of the mitochondria was not sufficient to induce apoptosis, although it enhanced TRAIL-induced apoptosis. Furthermore, oligomerization of Bak was partially responsible for the increased apoptosis in cells treated with both CGP and TRAIL. Thus, our results show that combination of an apoptogenic agent and an appropriate calcium channel blocker provide therapeutic advantages.
\end{abstract}

\section{Introduction}

The most common therapy for prostate cancer is androgen depletion, that is effective only on androgen-sensitive cells

Correspondence to: Dr M. Vijay Kumar, Research Department, Charlie Norwood VA Medical Center, 1 Freedom Way, Augusta, GA 30904, USA

E-mail: vijay.kumar2@va.gov

Key words: mitochondria, fission, calcium, prostate
(1-4), leaving very few options for the treatment of androgeninsensitive tumors. The goal of most cancer therapies, including antihormone therapy, is to induce apoptosis in tumor cells. Therefore, apoptosis-based therapies are being devised for both androgen-responsive and androgen-insensitive prostate cancer cells. Since its discovery in 1995, TRAIL (TNF $\alpha$ related apoptotic inducing ligand) has attracted enormous interest for the treatment of cancer $(5,6)$. TRAIL triggers apoptosis preferentially in tumor cells through the activation of specific death receptors DR4 (7) and DR5 $(5,6,8)$. Activation of death receptors by TRAIL results in the activation of procaspase- 8 , which in turn cleaves Bid. Translocation of truncated Bid to the mitochondria initiates apoptotic changes in the mitochondria, leading to oligomerization of Bax and/or Bak. Oligomerization of these proteins in the mitochondrial outer membrane (MOM) is considered a key step in mitochondriamediated apoptosis.

Alterations in mitochondrial milieu are correlated with apoptosis. One such event is the increase in ionic calcium $\left[\mathrm{Ca}^{2+}\right]$ concentration in the mitochondria. Finely tuned changes in cytosolic calcium $\left(\left[\mathrm{Ca}^{2+}\right]_{\mathrm{c}}\right)$ modulates a variety of intracellular functions, while disruption of $\mathrm{Ca}^{2+}$ handling leads to cell death. In resting cells, the levels of $\mathrm{Ca}^{2+}$ are low in the mitochondria, while the $\left[\mathrm{Ca}^{2+}\right]_{\mathrm{c}}$ levels are higher in apoptotic cells. Mitochondria uptake $\mathrm{Ca}^{2+}$ via an inner membrane channel, the Uniporter (9), and extrudes it to the cytoplasm through the mitochondrial $\mathrm{Na}^{+} / \mathrm{Ca}^{2+}$ exchanger $(10,11)$. Overload of $\mathrm{Ca}^{2+}$ in the mitochondria leads to their damage, affecting cellular function and survival (12). Increase in mitochondrial calcium $\left(\left[\mathrm{Ca}^{2+}\right]_{\mathrm{m}}\right)$ has been shown to induce mitochondria-mediated apoptosis as seen by alterations in mitochondrial membrane permeability (MMP), formation of the permeability transition pore (PTP), cytochrome c release, and activation of caspase- 9 and $-3(13,14)$.

Mitochondria undergo constant remodeling leading to changes in their shape and number. In apoptotic cells, the filamentous mitochondria characteristic of resting cells are pinched off into smaller organelles, which gives punctate appearance in a light microscope. This process is termed mitochondrial fission. In most physiological conditions, mitochondrial fusion (Mf) and mitochondrial fission (MF) are balanced, resulting in the formation of a tubular network of interconnected mitochondria. This thread-like architecture allows the rapid transmission of $\mathrm{Ca}^{2+}$ signals along the inter- 
connected mitochondria (15). Disruption of the mitochondrial network by excessive mitochondrial fission or impaired fusion is a common feature of apoptosis. Mitochondrial fission requires Dynamin related protein-1 (Drp1), an abundant cytoplasmic GTPase. Drp1 is regulated and sequestered by mitochondrial components under numerous apoptotic stimuli. At the mitochondria, Drp1 is recruited to mitochondrial outer membrane where it binds to Fis1, another key protein in mitochondrial fission that is anchored in the mitochondrial outer membrane $(16,17)$. Posttranslational modifications of Drp1, including phosphorylation, ubiquitination, and summoylation are critical in the process of mitochondrial fission. Functional impairment of Drp1 results in aggregates of large, interconnected mitochondria within cells (18). Furthermore, Drp1 is involved in promoting programmed cell death and its inhibition prevented mitochondrial division and Bax-mediated mitochondrial membrane permeabilization during apoptosis $(19,20)$.

We have previously shown that in prostate cancer cells increasing mitochondrial calcium, using the specific mitochondrial $\mathrm{Na}^{+} / \mathrm{Ca}^{2+}$ exchanger blocker, CGP37157 increased mitochondrial calcium and boosted the apoptotic effects of TRAIL (14). In the present study we demonstrate that mitochondrial calcium overload induced mitochondrial fission that is mediated through Drp1. Furthermore, inhibition of mitochondrial fission partially reduced the apoptotic effects of CGP and TRAIL.

\section{Materials and methods}

Cell culture and experimental design. Prostate cancer cell lines, DU145 (American Type Cell Culture, Manassas, VA) were maintained in RPMI-1640 (Hyclone, Logan, UT) containing $10 \%$ fetal bovine serum (Hyclone), $0.5 \%$ penicillin-streptomycin $(10,000 \mathrm{U} / \mathrm{ml}-\mu \mathrm{g} / \mathrm{ml})$, and $0.1 \%$ fungizone $(250 \mu \mathrm{g} / \mathrm{ml})$. Cells were seeded and incubated for 20-24 h to reach a confluency of $70-80 \%$. Cells were treated with fresh medium containing vehicle, $80 \mu \mathrm{M}$ benzothiazepin CGP-37157 (Calbiochem, San Diego, CA) and/or $50 \mathrm{ng} / \mathrm{ml}$ TRAIL (Biomol, Plymouth Meeting, PA). In drug combination groups TRAIL was added after 15 min of CGP pretreatment and incubated for $4 \mathrm{~h}$. In experiments where caspases were inhibited, $30 \mu \mathrm{M}$ pancaspase inhibitor, zVAD-FMK (R\&D Systems, Minneapolis, $\mathrm{MN}$ ) was added $30 \mathrm{~min}$ prior to drug treatment.

Transfection of prostate cancer cells. DU145 were transiently transfected with dominant negative Drp1 (Drp1-DN, K38A, a kind gift of Alexander van der Bliek, University of California, Los Angeles, CA) or BAK siRNA (synthesized by Dharmacon, Inc. Chicago, IL) using DMRIE-C (Invitrogen, Carlsbad, CA) as transfection reagent. The siRNA pool sense sequences used were CGACAUCAACCGACGCUAUUU, UAUGAGU ACUUCACCAAGAUU, GACGGCAGCUCGCCAUCAUUU and AAUCAUGACUCCCAAGGGUUU. Transfected cells were incubated for $8 \mathrm{~h}$, the medium was replaced with fresh medium, incubated for $36 \mathrm{~h}$ and were harvested and seeded in dishes for treatment.

For mitochondrial morphology experiments, electroporation was utilized for transfection. DU145 cells were resuspended in electroporation buffer $\left(120 \mathrm{mM} \mathrm{KCl}, 0.15 \mathrm{mM} \mathrm{CaCl}_{2}, 10 \mathrm{mM}\right.$
$\mathrm{K}_{2} \mathrm{HPO}_{4} / \mathrm{KH}_{2} \mathrm{PO}_{4}, 25 \mathrm{mM}$ HEPES, $2 \mathrm{mM}$ EGTA, $5 \mathrm{mM}$ $\mathrm{MgCl}_{2}, 50 \mathrm{mM}$ glutathione, $2 \mathrm{mM}$ ATP and $\mathrm{pH}$ 7.6) at a density of $10^{7}$ cells/ml. pAcGFP1-Mito (mito-green), a green fluorescent label for mitochondria construct (Clonetech Laboratories, Inc., Mountain View, CA), was added to 4 million cells in $0.4 \mathrm{ml}$. The mixture of cells and mito-green DNA were incubated for $5 \mathrm{~min}$ at RT, transferred to 4-mm cuvettes (BTX Inc., San Diego, CA) and subjected to electroporation pulses (3 pulses of $300 \mathrm{~V}$ for a period of $5 \mathrm{msec}$ each) using Electro Square Porator ${ }^{\mathrm{TM}}$ ECM 830 (BTX Inc.). Mixture of cells and DNA were incubated on ice for another $5 \mathrm{~min}$ before they were seeded in dishes and incubated for 24-48 h, at which time they were seeded in dishes for treatment and examination in fluorescent microscope (Zeiss Axioskop, Carl Zeiss, Inc., Thornwood, NY).

Measurement of fragmented mitochondria. To measure fragmented mitochondria two methods were utilized to stain the mitochondria: mitochondria were stained by histochemistry using Cox IV antibody (Cell Signaling Technology, Inc., Danvers, MA) or cells were transfected with mitogreen construct that targeted the expression of GFP to the mitochondria. Untransfected or mitogreen transfected DU145 cells were seeded on cover slips, treated as described above and the cells were examined on fluorescent microscope (Zeiss Axioskop, Carl Zeiss). Cells in which $<50 \%$ mitochondria are elongated were scored as cells containing fragmented mitochodnria. At least 5 fields ( $>100$ cells) per slide were counted in 4-5 experiments.

Protein extraction and Western blotting. Cells were harvested, washed with PBS and resuspended in lysis buffer (1X PBS, $1 \%$ Triton $\mathrm{X}-100,0.5 \%$ sodium deoxycholate, $0.1 \%$ SDS, $1 \mathrm{mM}$ EDTA, $0.5 \mu \mathrm{g} / \mu 1$ leupeptin, $1 \mu \mathrm{g} / \mu 1$ pepstatin, $1 \mu \mathrm{g} / \mu \mathrm{l}$ phenylmethyl sulfonyl fluoride and $1 \mu \mathrm{g} / \mathrm{ml}$ aprotinin). Cells were incubated on ice for $30 \mathrm{~min}$ and centrifuged at $10,000 \mathrm{~g}$ at $4^{\circ} \mathrm{C}$ for $10 \mathrm{~min}$. Protein concentration was estimated in the supernatant using Bio-Rad reagent (Bio-Rad Laboratories, Hercules, CA) and were analyzed by Western blots. The blots were stripped and re-probed with $\beta$-actin for loading control. The antibodies used in these experiments are specific to Bak (Cell Signaling Technology), Drp1 (Santa Cruz Biotechnology, Inc., Santa Cruz, CA, or Calbiochem, Gibbstown, NJ) and Fis1 (Alexis Biochemicals Corp., San Diego, CA).

Immunoprecipitation of Drp1. DU145 cells were treated as above and harvested in RIPA buffer (Millipore Corp. Billerica, MA). Equal amounts of proteins were incubated with Drp1 antibody (Santa Cruz Biotechnology) and protein-A/G agarose was added for protein precipitation. The beads were washed thrice, equal amount of proteins were separated by SDS PAGE and were processed for Western blots. The membranes were probed with a different Drp1 antibody (BD Biosciences, San Jose, CA) to reduce background noise.

Measurement of apoptosis. Apoptosis was measured using the M30 Apoptosense kit (DiaPharma Group, Inc., West Chester, OH). This is an ELISA using a specific antibody against a neoepitope of cytokeratin 18 that is generated by activated caspases. Apoptosense is highly sensitive and is 
specific to apoptosis. Apoptosense kit has been utilized successfully in our $(14,21,22)$, and other laboratories (23-25). Protein extract was added to 96 -well plates pre-coated with mouse monoclonal M30 antibody, horseradish peroxidase tracer solution was added to the wells and incubated for $4 \mathrm{~h}$. Color was developed by adding tetramethyl benzidine solution and the optical density was measured at $450 \mathrm{~nm}$ on a Spectra MAX340 microplate reader (Molecular Devices Corp., Sunnyvale, CA). Standard solution provided by the supplier was utilized for generating standard curves.

Measurement of intra-mitochondrial calcium. DU145 cells were incubated in dark with PBS containing 1\% BSA and $4 \mu \mathrm{mol} / 1$ Rhodamine 2-AM (Molecular Probes, Carlsbad, CA) for $40 \mathrm{~min}$. Pluronic F-127 (Molecular Probes), $5 \mu \mathrm{l} / \mathrm{ml}$ of $10 \%(\mathrm{w} / \mathrm{v})$ was added to the buffer to facilitate Rhodamine 2-AM loading. Cells were washed with PBS and incubated in Hank's balanced salt solution for $10 \mathrm{~min}$. For measurement of calcium, dishes were placed in a temperature-controlled $\left(37^{\circ} \mathrm{C}\right)$ Zeiss fluorescent microscope stage connected to a PTI ImageMaster monochromator (NJ, USA). Intensity of fluorescence was measured at 550-560 nm excitation and $580 \mathrm{~nm}$ emission wavelengths for Rhodamine 2-AM to measure mitochondrial calcium.

Immunocytochemistry of Drpl and Fis1. DU145 cells were cultured on cover slips for 20-24 h and treated with CGP and/or TRAIL as described above. Cells were fixed with $4 \%$ paraformaldehyde (Electron Microscopy Science, Hatfield, PA) and stained with COX IV antibody (Cell Signaling Technology) to enable visualization of mitochondrial shape (in zVAD-FMK pretreated cells and BAK siRNA transfection experiments). For the colocalization study, cells were treated with CGP and/or TRAIL then co-stained with Drp1 (BD Biosciences) and Fis1 antibody (Alexis Biochemicals Corp.). The two proteins were visualized using LSM 510 confocal microscope (Carl Zeiss).

Electron microscopy to visualize the mitochondrial structure. Cells were fixed overnight in $2 \%$ glutaraldehyde in $0.1 \mathrm{M}$ sodium cacodylate buffer, $\mathrm{pH} 7.4$, post-fixed in $2 \%$ osmium tetroxide and stained en bloc with $2 \%$ uranyl acetate. Cells were dehydrated embedded in Epon-Araldite resin. Thin sections were cut on a Leica EM UC6 ultramicrotome (Leica Microsystems, Inc., Bannockburn, IL) and stained with uranyl acetate and lead citrate. Sections were observed in a JEM 1230 transmission electron microscope (JEOL USA Inc., Peabody, MA).

Oligomerization of Bak. DU145 cells were lysed by disrupting with 27-gauge needles and centrifuged at $10,000 \mathrm{~g}$ at $4^{\circ} \mathrm{C}$ for $10 \mathrm{~min}$. Equal amount of cell lysates were incubated with $0.5 \mathrm{mM} / 1$ of the crosslinker bismaleimidohexane (Thermo Fisher Scientific, Rockford, IL) and incubated for $1 \mathrm{~h}$ at RT. Reaction mixture was quenched with $50 \mathrm{mM}$ dithiothreitol and incubated for another $15 \mathrm{~min}$ at RT. Samples were cleared from non-reactant crosslinker and EDTA using filters, Centricon YM-10 (Millipore Corp. Billerica, MA). Samples were separated by SDS PAGE, transferred to membrane and probed with Bak antibody.
Phosphorylation status of Drp1. DU145 cells were treated with vehicle or $80 \mu \mathrm{M}$ CGP for $2 \mathrm{~h}$. Cells were washed with ice-cold TBS (Tris pH 7.5 and $150 \mathrm{mM} \mathrm{NaCl}$ ) and incubated for $10 \mathrm{~min}$ in hypotonic buffer $(10 \mathrm{mM}$ Tris-base and $0.9 \%$ $\mathrm{NaCl}, 1 \mathrm{mM} \mathrm{MgCl}, 0.05 \% \mathrm{NP} 40,10 \%$ glycerol and freshly added DTT, PMSF and phosphatase inhibitors). Cells were centrifuged at 16,000 g for 20 min. Pellets (membranes, mitochondria and nuclear fractions) were washed $3 \mathrm{X}$ with the hypotonic buffer and incubated with lysis buffer (containing CHAPS and Benzonase) provided in the Phospho-Protein Purification Kit (Qiagen Inc., Valencia, CA). Equal amount of proteins were loaded on phospho-protein purification columns and phosphorylated proteins were eluted per manufacturer's suggestions. The eluted phosphorylated proteins were separated by SDS PAGE and probed with Drp1 antibody.

\section{Results}

Increasing mitochondrial calcium alters mitochondrial shape in prostate cancer cells. Our published data demonstrated that treatment of prostate cancer cells with CGP-37157 (CGP), a blocker of the mitochondrial $\mathrm{Na}^{+} / \mathrm{Ca}^{2+}$ exchanger increased the levels of mitochondrial calcium $\left(\left[\mathrm{Ca}^{2+}\right]_{\mathrm{m}}\right)$ but did not induce apoptosis (14). However, combining CGP with TRAIL induced highly significant apoptosis ( 25 -fold compared to controls). Furthermore, we demonstrated that mitochondria are critical in the induction of apoptosis by CGP and TRAIL. The goal of this study is to further examine the role of mitochondria in CGP/TRAIL-induced apoptosis.

As a first step, the effects of $\left[\mathrm{Ca}^{2+}\right]_{\mathrm{m}}$ overload on the mitochondrial fission in prostate cancer cells was examined. DU145 were transfected with mitogreen construct which is targeted for expression in the mitochondria. In control untreated cells the mitochondria are elongated filamentous structures (Fig. 1A). In CGP treated cells, mitochondria were punctate, pin-head-like structures typical of fragmented mitochondria. TRAIL by itself did not alter the structure of the mitochondria (Fig. 1A) though it induced apoptosis via mitochondrial pathway (14). The mitochondrial shape in CGP and TRAIL treated cells was similar to that of CGP treated cells, suggesting that the alterations in the mitochondrial shape were due to CGP, not TRAIL.

To confirm alterations in mitochondrial shape, cells were examined by electron microscopy. Micrographs (Fig. 1B) showed elongated mitochondria with clearly defined cristae in control cells. Elongated mitochondria were not seen in CGP treated cells. The mitochondria in these cells were rounded electron-dense structures. Thus, the punctuate shape observed in fluorescent microscope (Fig. 1A) seems to be fragmented mitochondria (Fig. 1B). Next, mitochondrial fragmentation in CGP treated cells were counted, which showed that CGP treatment induced $37 \%$ mitochondrial fragmentation by the first $60 \mathrm{~min}$, which increased to $51 \%$ in $2 \mathrm{~h}$ and $60 \%$ by $4 \mathrm{~h}$ (Fig. 1C). Therefore, all further experiments were conducted with CGP treatment for $4 \mathrm{~h}$.

The above results showed mitochondrial fragmentation by CGP, while our earlier report demonstrated increased mitochondrial calcium in CGP treated cells (14). Therefore, it was of interest to determine whether $\mathrm{Ca}^{2+}$ is still associated with fragmented mitochondria. Mitochondrial calcium was 
A

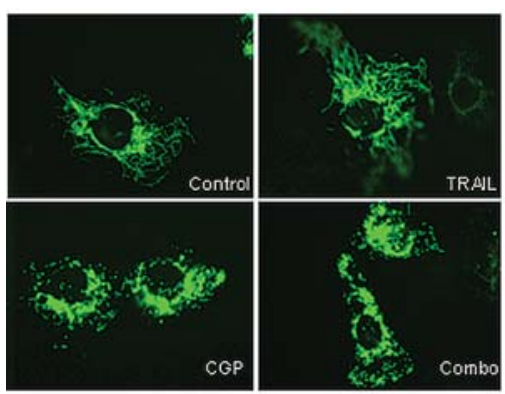

C

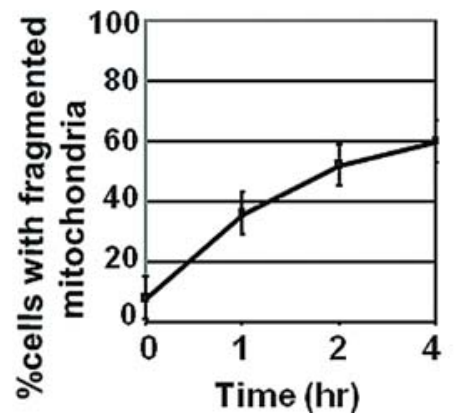

B
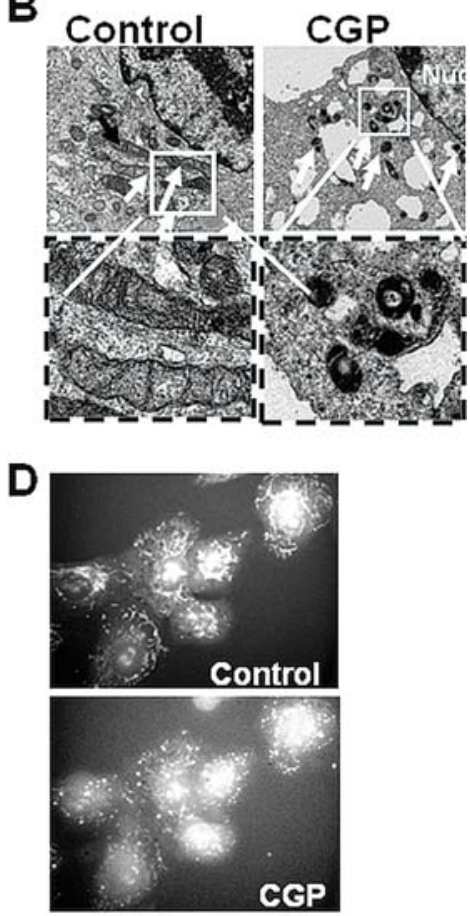

Figure 1. Enhanced mitochondrial calcium induced mitochondrial fragementation in prostate cancer cells. (A) Prostate cancer cells, DU145, were transfected with mitogreen construct which is targeted for expression in the mitochondria. These cells were treated with $80 \mu$ mol/l of benzothiazepin CGP-37157 for $4 \mathrm{~h}$ and $15 \mathrm{~min}$ (CGP) or with $50 \mathrm{\eta g} / \mathrm{ml}$ TRAIL for $4 \mathrm{~h}$. In combination groups (combo) TRAIL was added after 15 min of CGP pretreatment and incubated for $4 \mathrm{~h}$. The shape of the mitochondria was examined under a fluorescent microscope. This is a representative photograph of one of the four separate experiments. Mitochondria are green thread-like structures in the control and TRAIL-treated cells, while it is dot-like or puntate in CGP and CGP/TRAIL treated cells. (B) Cells were treated with vehicle (control) or CGP and processed for transmission electron microscopy as described in Materials and methods. Top panels show lower magnification pictures, while the bottom panels show enlargement of specified areas. Elongated mitochondria (solid arrows), while fragmented mitochondria are shown as stippled arrows. (C) Cells were treated with $60 \mu \mathrm{M}$ CGP and the presence of fragemented mitochondria in 3-4 fields were scored after 1,2 , and $4 \mathrm{~h}$. The values are mean \pm SD of at least three experiments. (D) DU145 cells in culture were loaded with Rhodamine 2-AM and incubated for $40 \mathrm{~min}$. Cells were observed using a Zeiss fluorescent microscope connected to a PTI Image Master monochromator. Intensity of fluorescence in the mitochondria was measured at 550-560 nm excitation and $580 \mathrm{~nm}$ emission wavelengths for Rhodamine 2-AM to measure calcium. Cells were under continuous monitoring. Photomicrographs of the same group of cells were taken before (control) and after treatment with CGP. This is a representative photograph of one of the four separate experiments. Calcium in the mitochondria can be seen as fluorescent dots.

A
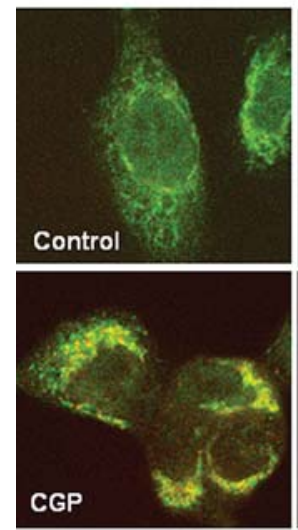
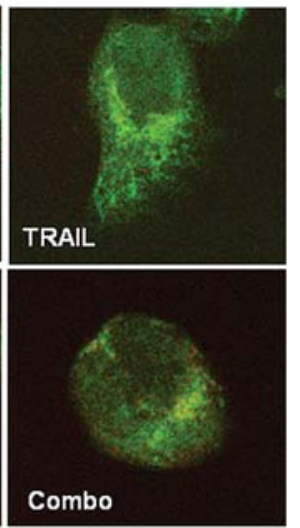

B

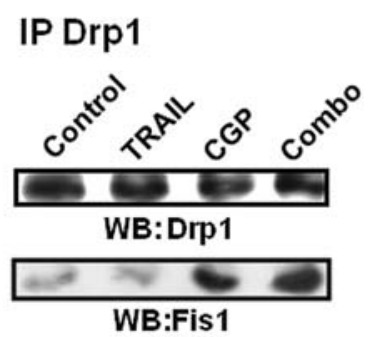

C

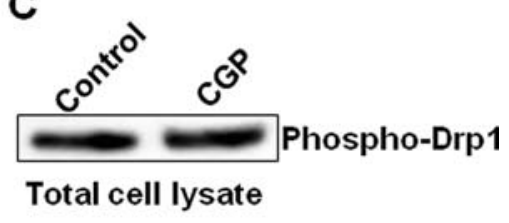

Phospho-Drp1

Mitochondrial fraction

Figure 2. CGP induced interaction between Drp1 and Fis1 that are required for fragmentation of the mitochondria. (A) DU145 cells were treated as described in Fig. 1. Cells were processed for immunocytochemistry using specific antibodies for Drp1 (red fluorescence) and Fis1 (green fluorescence). Images were examined using a confocal microscope. Yellow fluorescence indicates the site of interaction between Drp1 and Fis1. (B) Drp1 was immunoprecipitated in total cell extracts from control and treated cells. The immunoprecipitated proteins were separated and the Western blots were probed with Drp1 or Fis1 antibodies. (C) For investigating the phosphorylation status of Drp1, total cell lysates and mitochondrial fractions were utilized. The proteins were separated using phospho-protein purification kit, separated on gels and Western blots were probed with Drp1 antibody. 
A

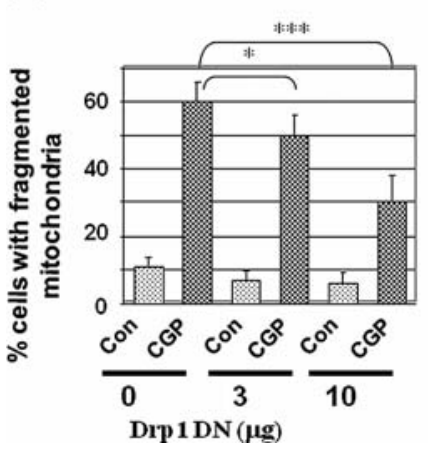

B

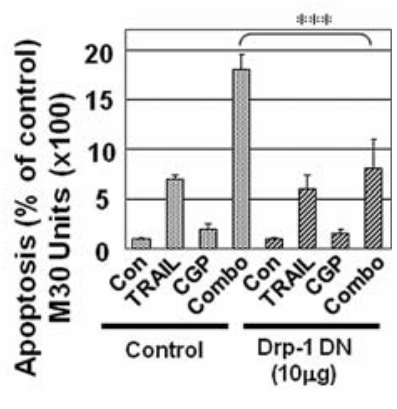

Figure 3. Drp1 down-regulation mitigated CGP/TRAIL-induced apoptosis and CGP-induced mitochondrial fission. (A) DU145 cells were transfected with 3 or $10 \mu \mathrm{g}$ Drp1 dominant negative (Drp1-DN) construct and were treated with $60 \mu \mathrm{M}$ CGP. The number of cells with fragmented mitochondria were counted as described earlier and were expressed as percentage of cells with fragmented mitochondria to total cells. The values are mean $\pm \mathrm{SD}$ of at least three experiments. ${ }^{*} \mathrm{P}<0.05 ;{ }^{* * *} \mathrm{P}<0.001$. (B) Cells were transfected with $10 \mu \mathrm{g}$ Drp1-DN construct and apoptosis was measured using M30 Apoptosense kit. The data are expressed as M30 antigen $\mathrm{U} / \mu \mathrm{g}$ protein compared with control. The values are mean $\pm \mathrm{SD}$ of at least three experiments. ${ }^{* * *} \mathrm{P}<0.001$

visualized by Rhodamine 2-AM, which demonstrated that in control cells, $\mathrm{Ca}^{2+}$ was distributed throughout the elongated mitochondria (Fig. 1D). Interestingly, $\mathrm{Ca}^{2+}$ continued to be associated with punctate/fragmented mitochondria in treated cells.

CGP induces interaction between Drpl and Fisl that is required for fragmentation of the mitochondria. The cytoplasmic Drp1 and the mitochondrial Fis1 are critical proteins in mitochondrial fragmentation. To examine their role in CGP/TRAIL-induced mitochondrial fragmentation and apoptosis, cells were stained for the presence of Drp1 and Fis1. In control cells, Drp1 (red fluorescence) was not readily seen probably because it is uniformly diffuse in the cytoplasm (Fig. 2A). On the other hand, the mitochondrial Fis 1 (green fluorescence) depicted the location of mitochondria. In CGP treated cells, in addition to green fluorescence of Fis1 high levels of yellow fluorescence was observed that is indicative of interactions between Drp1 (red fluorescence) and Fis1 (green) proteins. The distribution of proteins in TRAIL treated cells were similar to that of controls, while the cells treated with both CGP and TRAIL were similar to CGP-treated cells. These results suggest that interactions between Drp1 and Fis 1 correlated with mitochondrial fragmentation in CGP treated cells.

To confirm these interactions, proteins were immunoprecipitated using Drp1-specific antibody and the presence of Drp1 and Fis1 proteins were examined by Western blotting. Drp1 is abundant in DU145 and its expression was not altered by treatment with CGP and TRAIL alone or together (Fig. 2B). As expected (Fig. 2A), treatment with CGP alone or together with TRAIL resulted in significant band for Fis 1 protein, confirming the interactions between Drp1 and Fis1 (Fig. 2B). It has been shown that phosphorylation and dephosphorylation of Drp1 at specific sites, play an important role in its conformational change and translocation to the mitochondria, which would lead to interaction with Fis1. In the present study, we found that Drp1 is readily phosphorylated in the cells as noted by similar levels of phosphorylation in total cell lysates, in both vehicle and CGP-treated cells (Fig. 2C). In the mitochondrial fraction, the translocated Drp1 under CGP treatment was still phosphorylated (Fig. 2C), confirming the translocation of Drp1 to the mitochondria upon CGP treatment (Fig. 2A).

Drpl down-regulation mitigates CGP/TRAIL-induced apoptosis and CGP-induced mitochondrial fission. To confirm the role of Drp1, DU145 cells were transfected with dominant negative Drp1 (Drp1-DN) expression vector, which resulted in dose-dependent decrease in mitochondrial fragmentation (Fig. 3A). Next, we examined whether inhibiting Drp1 altered apoptotic response. Cells were treated with CGP and TRAIL and apoptosis was measured. As demonstrated earlier (14), treatment with both CGP and TRAIL significantly increased apoptosis in cells that were not transfected with DN-Drp1 (Fig. 3B). However, cells transfected with Drp1-DN, responded less to the apoptotic effect of CGP/TRAIL when compared to untransfected cells, suggesting that Drp1 is required for the induction of apoptosis in these cells.

Pro-apoptotic protein BAK is involved in CGP/TRAIL-induced apoptosis. $\mathrm{Bcl} 2$ family proteins have been shown to play a major role in mitochondria-mediated apoptosis. To determine whether these proteins are involved in CGP/TRAIL-induced mitochondrial fragmentation and apoptosis, we first examined the expression of Bax and Bcl2 in DU145 cells. As shown in Fig. 4A, Bax and Bcl2 were not expressed in DU145 cells, while Bak was expressed (Fig. 4B). Therefore, we examined the role of Bak as: i) Bak is an equally potent pro-apoptotic protein, ii) Bak is known to induce apoptosis even in the absence of Bax and, iii) we have recently demonstrated that the presence of Bak is critical for the fragmentation of the mitochondria (26). Immunoblots showed that TRAIL induced the formation of Bak dimers (Fig. 4B), while Bak dimerization was not seen in CGP treated cells. Combination of CGP and TRAIL induced significant increase in dimerized Bak. These results suggest that TRAIL is responsible for Bak oligomerization not CGP. Next, to inhibit the levels of Bak, cells were transfected with increasing concentrations of Bak siRNA, which showed that even $10 \eta \mathrm{M}$ Bak siRNA was effective in inhibiting the expression of Bak (Fig. 4C). Measurement of apoptosis in cells where Bak expression is inhibited by siRNA, reduced TRAIL-induced apoptosis compared to cells expressing WT Bak. These results show that Bak facilitated, at least partially, TRAIL-induced apoptosis. However, absence of Bak did not affect mitochondrial fragmentation in DU145 cells transfected with Bak siRNA (Fig. 4E) indicating that Bak is not required for CGP-induced fragmentation of the mitochondria. Thus, CGP induces fragmentation, but does not induce apoptosis, while TRAIL does not induce fragmentation, but induces Bak-mediated apoptosis.

Requirement of caspases in CGP/TRAIL-induced apoptosis and mitochondrial fragmentation. We and others have shown that induction of apoptosis by TRAIL requires the activation 
A

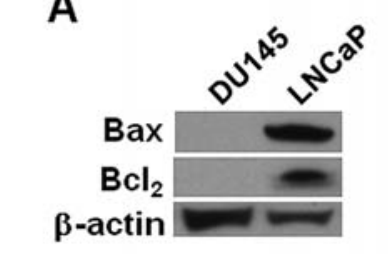

B
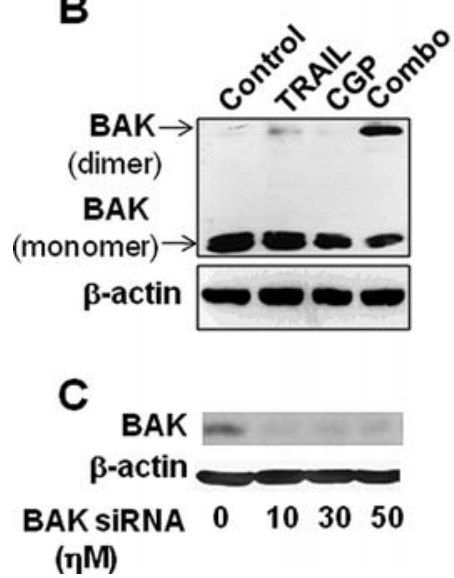

D

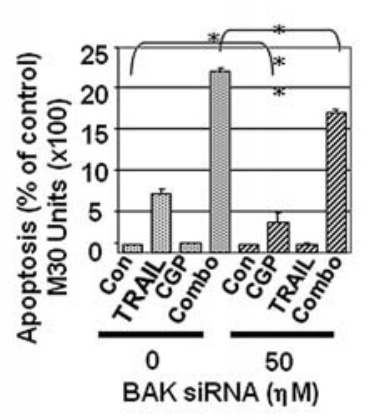

$\mathbf{E}^{-}$
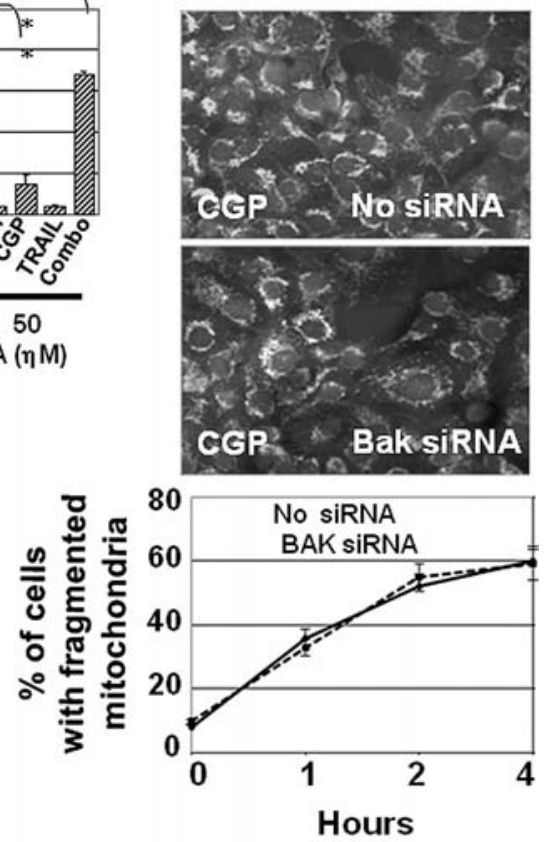

Figure 4. Involvement of BAK in CGP/TRAIL-induced apoptosis. (A) Proteins were extracted from prostate cancer cells DU145 and LNCaP, and were analyzed by Western blotting to examine the presence of $\mathrm{Bax}$ and $\mathrm{BCl} 2$ proteins. (B) To examine the dimerization of Bak, cells were treated with CGP and/or TRAIL as described. Cells were lysed and incubated with the cross-linker BMH and analyzed by Western blotting. The monomer Bak was present as a $28-\mathrm{kDa}$ protein, while the dimer is $\sim 56 \mathrm{kDa}$. (C) To inhibit the expression of Bak, cells were transfected with 10,30 , and $50 \eta \mathrm{M}$ BAK siRNA and the protein extracts were analyzed by Western blots for the presence of Bak or the loading control B-actin. (D) Cells were transfected with $50 \eta \mathrm{M}$ BAK siRNA and treated with CGP and TRAIL as described above. Apoptosis was measured using M30 Apoptosense kit and is expressed as M30 antigen U/ $\mu$ g protein compared with control. The values are mean $\pm \mathrm{SD}$ of at least three experiments. ${ }^{*} \mathrm{P}<0.05 ;{ }^{* * *} \mathrm{P}<0.001$. (E) Cells were transfected with $50 \eta \mathrm{M} \mathrm{BAK}$ siRNA then treated with $60 \mu \mathrm{M}$ CGP for 1,2, and $4 \mathrm{~h}$. The fragmentation of the mitochondria were scored and expressed as percentage of cells that showed fragmented mitochondria in the presence or absence of Bak. The values are mean \pm SD of at least three experiments (bottom panel). The top panels are photomicrographs of cells showing the fragmentation of mitochondria in cells with or without Bak expression.

A

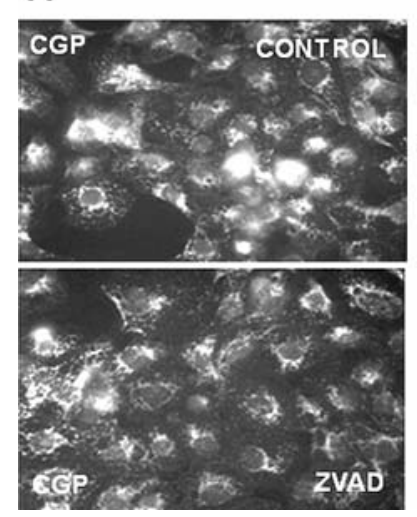

B

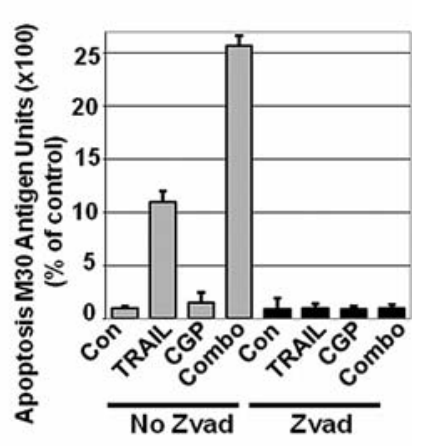

Figure 5. Caspases are involved in apoptosis induced by CGP but not in mitochondrial fission. (A) DU145 cells were pretreated with the pan-caspase inhibitor zVAD-FMK and treated as described. Fragmented mitochondria were examined in a fluorescent microscope. (B) Cell extracts were utilized to measure apoptosis using M30 Apoptosense kit and is expressed as M30 antigen $\mathrm{U} / \mu \mathrm{g}$ protein compared with control. The values are mean $\pm \mathrm{SD}$ of at least three experiments.

of caspases. To examine the requirement of caspases in mitochondrial fragmentation, caspases were inhibited by pan-caspase inhibitor zVAD-FMK and the mitochondrial fragmentation was scored. Results showed that inhibition of caspase activation did not affect CGP-induced mitochondrial fragmentation (Fig. 5A). However, in the absence of caspases, CGP and TRAIL (either individually or together) did not induce apoptosis (Fig. 5B). These results suggest that fragmentation of the mitochondria did not require caspases, although induction of apoptosis was caspase mediated.

\section{Discussion}

Mitochondria play a critical role in cellular metabolism as an energy source and for maintaining calcium homeostasis. Recently, the changes in mitochondrial shape were correlated with mitochondria-mediated apoptosis. Elongated, filamentous mitochondria are associated with resting, non-apoptotic cells. Upon apoptotic stimulus, the filamentous mitochondria undergo a process of 'fission' whereby the mitochondria are pinched and segregated into smaller punctate or fragmented moieties (27-32).

Calcium signaling mechanisms govern a multitude of vital cell functions that affect cell survival. Disruption of intracellular $\mathrm{Ca}^{2+}$ is a powerful activator of multiple cell damaging processes, such as apoptosis or necrosis $(33,34)$. Earlier, we observed that increasing the concentration of mitochondrial $\mathrm{Ca}^{2+}$ by inhibiting its efflux from the mitochondria facilitated apoptosis induced by TRAIL (14). Furthermore, local increase in $\left[\mathrm{Ca}^{2+}\right]_{\mathrm{m}}$ altered the mitochondrial 
membrane potential and increased efflux of cytochrome c from the mitochondria (14). Here, we examined the effects of these changes on the shape of the mitochondria, which has been correlated to altered function of the organelle and death of the cell. Our results demonstrated that CGP induced mitochondrial fragmentation. Thus, increase in $\left[\mathrm{Ca}^{2+}\right]_{\mathrm{m}}$ may be responsible for mitochondrial fission. More importantly, unlike published studies where $\left[\mathrm{Ca}^{2+}\right]_{\mathrm{m}}$ was elevated by increasing total calcium load in the cell, in these experiments the $\left[\mathrm{Ca}^{2+}\right]_{\mathrm{m}}$ was elevated by specifically blocking the exit of $\mathrm{Ca}^{2+}$ from the mitochondria.

Mitochondrial fission requires the translocation of the cytoplasmic Drp1 to the mitochondria and its interaction with the mitochondrial protein Fis1 $(17,35)$. In apoptotic cells, mitochondrial membrane depolarization facilitates the accessibility of the mitochondrial Fis1 to Drp1 (36). The interaction between Drp1 and Fis1 is deemed critical for the mitochondrial fragmentation $(18,28,37,38)$. Furthermore, site specific phosphorylation and dephosphorylation of Drp1 seems to be a requirement for the interaction of Drp1 and Fis1 (39). We have demonstrated interactions between Drp1 and Fis1 using cytochemistry and immunoprecipitations techniques (Fig. 2A and B). Surprisingly, we did not notice change of the total phosphorylation status between CGP treated and control cells in total cell lysates. In mitochondrial fractions Drp1 was present under CGP-treatment in phosphorylated condition (Fig. 2C). Finally, transfection of DN-Drp1 construct significantly reduced CGP-induced mitochondrial fragmention (Fig. 3A). Thus, CGP-induced mitochondrial fragmentation requires functional Drp1 leading to the interaction between Drp1 and Fis1.

In the past, we have demonstrated that TRAIL induced apoptosis in prostate cancer cells by activating caspase-8, which in turn was responsible for truncation of the proapoptotic protein Bid (14). Truncated Bid (tBid) translocated to the mitochondria leading to series of mitochondrial apoptotic events. It is interesting that CGP and TRAIL seem to affect the mitochondria in distinctly separate pathways. On the one hand, as mentioned earlier, CGP increased $\left[\mathrm{Ca}^{2+}\right]_{\mathrm{m}}$ leading to alterations in the mitochondria resulting in the availability of Fis1 for recruitment of Drp1 and fragmentation of the mitochondria. On the other hand, TRAIL induced the involvement of pro-apoptotic members of the Bcl2 family (e.g., tBid and Bak) in inducing mitochondria-mediated apoptosis. Activation of both these pathways (in CGP and TRAIL treated cells) results in highly synergistic induction of apoptosis. We suggest that the increased apoptosis is due to priming of the mitochondria by the CGP. This hypothesis is supported by the decrease in apoptotic response to combination therapy in cells transfected with DN-Drp1 (Fig. 3B) suggesting that CGP-induced events precede those of TRAIL-induced events. It is interesting that TRAIL-induced events do not have any influence of CGP-induced changes such as fragmentation. For example, inhibiting the expression of Bak did not affect CGP-induced fragmentation, while TRAIL-induced apoptosis was reduced (Fig. 4D and E).

In conclusion, in this report we demonstrate that specifically increasing mitochondrial calcium led to mitochondrial fragmentation. Induction of mitochondrial fragmentation facilitated increase in apoptosis induced by TRAIL. Altered mitochondrial events including the interactions between Drp1 and Fis1, dimerization of Bak, changes in the mitochondrial membrane potential, formation of permeability transition pore and other events are responsible for CGP/TRAIL induced apoptosis. Thus, these results suggest that a combination of an apoptogenic agent such as TRAIL and a calcium channel blocker, present therapeutic advantages. As these drugs are effective at the level of the mitochondria this therapeutic approach provides specificity and low toxicity compared to cytotoxic drugs that are presently being utilized in therapy.

\section{Acknowledgements}

This research was funded by a VA Merit Review grant to Dr M. Vijay Kumar.

\section{References}

1. Bruckheimer EM, Gjertsen BT and McDonnell TJ: Implications of cell death regulation in the pathogenesis and treatment of prostate cancer. Semin Oncol 26: 382-398, 1999.

2. Colombel MC and Buttyan R: Hormonal control of apoptosis: the rat prostate gland as a model system. Methods Cell Biol 46: 369-385, 1995.

3. Labrie F, Dupont A, Belanger A, et al: New approach in the treatment of prostate cancer: Complete instead of partial withdrawal of androgens. Prostate 4: 579-594, 1983.

4. Santen RJ: Clinical review 37: endocrine treatment of prostate cancer. J Clin Endocrinol Metab 75: 685-689, 1992.

5. Sheridan JP, Marsters SA, Pitti RM, et al: Control of TRAILinduced apoptosis by a family of signaling and decoy receptors. Science 277: 818-821, 1997

6. Walczak H, Degli-Esposti MA, Johnson RS, et al: TRAIL-R2: a novel apoptosis-mediating receptor for TRAIL. EMBO J 16: 5386-5397, 1997.

7. Pan G, O'Rourke K, Chinnaiyan AM, Gentz R, Ebner R, Ni J and Dixit VM: The receptor for the cytotoxic Ligand TRAIL. Science 276: 111-113, 1997

8. Pan G, Ni J, Wei Y-F, Yu G-1, Gentz R and Dixit VM: An antagonist decoy receptor and a death domain-containing receptor for TRAIL. Science 277: 815-818, 1997.

9. Gunter TE, Buntinas L, Sparagna G, Eliseev R and Gunter K: Mitochondrial calcium transport: mechanisms and functions. Cell Calcium 28: 285-296, 2000.

10. Cox DA and Matlib MA: A role for the mitochondrial $\mathrm{Na}(+)-$ $\mathrm{Ca}^{2+}$ exchanger in the regulation of oxidative phosphorylation in isolated heart mitochondria. J Biol Chem 268: 938-947, 1993.

11. White RJ and Reynolds IJ: Mitochondrial depolarization in glutamate-stimulated neurons: an early signal specific to excitotoxin exposure. J Neurosci 16: 5688-5697, 1996.

12. Berridge MJ, Lipp P and Bootman MD: The versatility and universality of calcium signalling. Nat Rev Mol Cell Biol 1: 11-21, 2000.

13. Hunter DR, Haworth RA and Southard JH: Relationship between configuration, function, and permeability in calcium-treated mitochondria. J Biol Chem 251: 5069-5077, 1976.

14. Kaddour-Djebbar I, Lakshmikanthan V, Shirley RB, Ma Y, Lewis RW and Kumar MV: Therapeutic advantage of combining calcium channel blockers and TRAIL in prostate cancer. Mol Cancer Ther 5: 1958-1966, 2006.

15. Ichas F, Jouaville LS and Mazat J-P: Mitochondria are excitable organelles capable of generating and conveying electrical and calcium signals. Cell 89: 1145-1153, 1997.

16. Suzuki M, Jeong S-Y, Karbowski M, Youle RJ and Tjandra N: The solution structure of human mitochondria fission protein Fis1 reveals a novel TPR-like helix bundle. J Mol Biol 334: 445-458, 2003.

17. Yoon Y, Krueger EW, Oswald BJ and McNiven MA: The mitochondrial protein hFis 1 regulates mitochondrial fission in mammalian cells through an interaction with the dynamin-like protein DLP1. Mol Cell Biol 23: 5409-5420, 2003.

18. Smirnova E, Griparic L, Shurland D-L and van der Bliek AM: Dynamin-related protein Drp1 is required for mitochondrial division in mammalian cells. Mol Biol Cell 12: 2245-2256, 2001. 
19. Cassidy-Stone A, Chipuk JE, Ingerman E, et al: Chemical inhibition of the mitochondrial division dynamin reveals its role in bax/bak-dependent mitochondrial outer membrane permeabilization. Dev Cell 14: 193-204, 2008.

20. Karbowski M: Spatial and temporal association of Bax with mitochondrial fission sites, Drp1, and Mfn2 during apoptosis. J Cell Biol 159: 931-938, 2002.

21. Lakshmi RVand Basu BJ: Fabrication of superhydrophobic sol-gel composite films using hydrophobically modified colloidal zinc hydroxide. J Colloid Interface Sci 339: 454-460, 2009.

22. Shirley RB, Kaddour-Djebbar I, Patel DM, Lakshmikanthan V, Lewis RW and Kumar MV: Combination of proteasomal inhibitors lactacystin and MG132 induced synergistic apoptosis in prostate cancer cells. Neoplasia 7: 1104-1111, 2005.

23. Cummings J, Hodgkinson C, Odedra R, et al: Preclinical evaluation of M30 and M65 ELISAs as biomarkers of drug induced tumor cell death and antitumor activity. Mol Cancer Ther 7: 455-463, 2008.

24. Greystoke A, Cummings J, Ward T, et al: Optimisation of circulating biomarkers of cell death for routine clinical use. Ann Oncol 19: 990-995, 2008.

25. Steele NL, Plumb JA, Vidal L, et al: A phase 1 pharmacokinetic and pharmacodynamic study of the histone deacetylase inhibitor belinostat in patients with advanced solid tumors. Clin Cancer Res 14: 804-810, 2008.

26. Brooks C, Wei Q, Feng L, Dong G, Tao Y, Mei L, Xie Z-J and Dong Z: Bak regulates mitochondrial morphology and pathology during apoptosis by interacting with mitofusins. Proc Natl Acad Sci USA 104: 11649-11654, 2007.

27. Bossy-Wetzel E, Barsoum MJ, Godzik A, Schwarzenbacher R and Lipton SA: Mitochondrial fission in apoptosis, neurodegeneration and aging. Curr Opin Cell Biol 15: 706-716, 2003.

28. Chen $\mathrm{H}$ and Chan DC: Emerging functions of mammalian mitochondrial fusion and fission. Hum Mol Genet 14: R283-R289, 2005 .
29. Frank S: The role of dynamin-related protein 1 , a mediator of mitochondrial fission, in apoptosis. Dev Cell 1: 515-525, 2001.

30. Hom JR, Gewandter JS, Michael L, Sheu S-S and Yoon Y: Thapsigargin induces biphasic fragmentation of mitochondria through calcium-mediated mitochondrial fission and apoptosis. J Cell Physiol 212: 498-508, 2007.

31. Lee Y-J, Jeong S-Y, Karbowski M, Smith CL and Youle RJ: Roles of the mammalian mitochondrial fission and fusion mediators Fis1, Drp1, and Opa1 in apoptosis. Mol Cell Biol 15: 5001-5011, 2004.

32. Youle RJ and Karbowski M: Mitochondrial fission in apoptosis. Nat Rev Mol Cell Biol 6: 657-663, 2005.

33. D'Herde K and Leybaert L: Intracellular free calcium related to apoptotic cell death in quail granulosa cell sheets kept in serum-free culture. Cell Death Differ 4: 59, 1997.

34. Trump BF and Berezesky IK: Calcium-mediated cell injury and cell death. FASEB J 9: 219-228, 1995.

35. Jagasia R, Grote P, Westermann B and Conradt B: DRP-1mediated mitochondrial fragmentation during EGL-1-induced cell death in C. elegans. Nature 433: 754-760, 2005.

36. Yu T, Fox RJ, Burwell LS and Yoon Y: Regulation of mitochondrial fission and apoptosis by the mitochondrial outer membrane protein hFis1. J Cell Sci 118: 4141-4151, 2005.

37. Shaw JM and Nunnari J: Mitochondrial dynamics and division in budding yeast. Trends Cell Biol 12: 178-184, 2002.

38. Yoon Y, Pitts KR and McNiven MA: Mammalian dynamin-like protein DLP1 tubulates membranes. Mol Biol Cell 12: 2894-2905, 2001.

39. Han X-J, Lu Y-F, Li S-A, et al: CaM kinase I alpha-induced phosphorylation of Drp1 regulates mitochondrial morphology. J Cell Biol 182: 573-585, 2008. 